\title{
Biparental inheritance of mitochondrial DNA revisited
}

Alistair T. Pagnamenta ${ }^{1}$, Wei Wei ${ }^{2}$, Shamima Rahman ${ }^{3}$ and Patrick F. Chinnery ${ }^{2+}$

${ }^{1}$ Wellcome Centre for Human Genetics and the NIHR Oxford Biomedical Research Centre, University of Oxford, Oxford, UK

${ }^{2}$ Department of Clinical Neurosciences and the MRC Mitochondrial Biology Unit, University of Cambridge, Cambridge Biomedical Campus, Cambridge, UK

${ }^{3}$ UCL Great Ormond Street Institute of Child Health, London, UK

†e-mail:pfc25@cam.ac.uk

\section{Orcid IDs}

ATP: https://orcid.org/0000-0001-7334-0602

WW: https://orcid.org/0000-0002-2945-3543

SR: https://orcid.org/0000-0003-2088-730X

PFC: https://orcid.org/0000-0002-7065-6617

\section{Standfirst}

Evidence for a biparental mode of mitochondrial DNA (mtDNA) inheritance has been sparse and remains controversial. Recent studies using a range of complementary techniques do not support paternal transmission of mtDNA, and highlight the co-amplification of rare, concatenated nuclear mtDNA segments as a technical artefact that may explain previous observations.

First demonstrated in humans in $1980^{1}$, strict maternal inheritance of mitochondrial DNA (mtDNA) has stood the test of time, with the only counterexample ${ }^{2}$, a single case report suggesting paternal mtDNA inheritance, never having been convincingly replicated. Uniparental inheritance enables rapid cytoplasmic genome evolution, contributing to extensive polymorphism in the extant population. This has been exploited by population geneticists to map human migration throughout history, and to match biological samples in forensic science. Most human cells contain $>100$ copies of mtDNA, and a mixed mtDNA population in the same individual is termed 'heteroplasmy'. Precise heteroplasmy levels change rapidly upon transmission to offspring owing to a genetic bottleneck during oogenesis. Thus, novel variants are either lost or progress to fixation within a few generations, explaining 
why high heteroplasmy levels are uncommon in the population. In 2018, a controversial study describing high levels of heteroplasmy in three multigenerational families proposed a previously unrecognized biparental form of mtDNA inheritance ${ }^{3}$. However, newly published data obtained using a range of complementary techniques do not support paternal transmission and suggest a more parsimonious explanation (Supplementary Table 1).

\section{Challenging the maternal paradigm}

Using a combination of long-range PCR amplification and next generation sequencing, Luo et al. identified three large families where multiple individuals apparently harboured an unusually high number of heteroplasmic mtDNA variants ${ }^{3}$ (referred to by others as 'multiHets'). MtDNA haplotype segregation studies persuaded the authors that biparental inheritance of mtDNA had occurred in these families. Unlike most previously published pedigrees, remarkably consistent levels of heteroplasmy were seen across multiple generations, and the same 'heteroplasmic haplotype' was consistently transmitted at high levels (24-76\% haplotype frequency) within each ostensibly unrelated family. These findings were widely discussed in social and mainstream media, prompting commentaries either extolling the new discovery 4 or expressing sceptical viewpoints, pointing out the need for orthogonal proof before rejecting an established dogma ${ }^{5}$.

Several technical explanations were proposed to explain the apparent 'biparental transmission', including that the study had detected segments of mtDNA embedded within the nuclear genome, so-called NUclear-MiTochondrial DNA segments (NUMTs). Luo et al. subsequently counter-argued that the linearized nature of these sequences would have rendered them undetectable to long-range PCR using outward-facing primers. For single-copy NUMTs, the contaminating haplotype would also be diluted by genuine mtDNA to extremely low levels, well below reported values. However, if the sequence integrated into a nuclear chromosome involved a number of concatenated mtDNA repeats (termed a "Mega-NUMT"6), this could both allow PCR-amplification and also lead to high levels of a mixed haplotype.

\section{Prevalence of multiHet individuals}

An initial attempt to replicate Luo et al.'s findings using whole-genome sequencing (WGS) did not detect any signatures of biparental mtDNA inheritance in 41 families $^{7}$. A larger WGS study in 11,035 parent-child trios excluded sample contamination and validated familial 
relationships using autosomal SNPs ${ }^{8}$. A signature resembling biparental mtDNA inheritance was seen in 7 families, with the paternal haplotype at allelic fractions of 5-25\%. However, the paternally inherited haplotypes were transmitted only to half of the offspring, and an equal number were maternally transmitted, suggesting nuclear DNA transmission. Wei et al. used split-read analyses to identify rare or unique NUMTs transmitted from the father in all 7 families with the biparental inheritance signature ${ }^{8}$. Five different integration sites were detected, all at non-coding loci. Split-reads mapping to different segments of mtDNA were consistent with Mega-NUMTs, and two integration sites shared between unrelated families were thought to represent ancestral Mega-NUMTs.

Subsequently, Lutz-Bonengel et al. reported similar, albeit coincidental, findings based on a single individual who was a forensics laboratory trainee ${ }^{9}$, mapping a Mega-NUMT integration site to $14 q 31$ using fluorescence in situ hybridization. DNA analysis of 19 other family members using a variety of tissues (bone DNA extraction using grave site samples were even obtained for deceased grandparents) showed mixed haplotypes in several individuals, again consistent with nuclear DNA transmission.

Finally, Bai et al. searched for multiHet individuals with $\geq 5$ heteroplasmic mtDNA variants that appeared to have heteroplasmy levels of 10-90\%; this study investigated only individuals with no previous blood transfusions or organ transplants, and validated their findings in an independent blood sample ${ }^{10}$. Exome and PCR-amplified mtDNA sequencing identified the multiHet phenomenon in 104 out of 27,388 individuals. The difference in incidence likely reflects differences in ascertainment, sequencing techniques and bioinformatic pipelines. Their findings confirmed that the phenomenon is rare - and likely discounted in many laboratories as suspected sample contamination.

\section{Inverse correlation with mtDNA content}

In tissues with high energy demands, such as muscle, cells generally have a greater ratio of mtDNA to nuclear DNA. If the heteroplasmy signature were due to Mega-NUMTs rather than bona fide biparental inheritance, an inverse correlation between the Mega-NUMT haplotype fraction and mtDNA content would be expected. Comparing DNA from buccal cells and muscle, the anticipated dilution effect due to higher mtDNA content in muscle was clearly observed $^{9,10}$. A similar signature was also seen in blood samples, where mtDNA levels reflect the composition of different cell types in whole blood ${ }^{8}$. Hair shaft analysis is often used in 
forensic sciences; DNA from this source contains only minute amounts of highly fragmented nuclear DNA. Similarly, thrombocytes are a type of blood cell known to lack a nucleus. LutzBonengel et al. demonstrated loss of the Mega-NUMT heteroplasmy signature in both of these sources of DNA ${ }^{9}$. Conversely, progressively depleting mtDNA from cells led to a predominance of the Mega-NUMT associated haplotype ${ }^{9}$.

\section{Clinical relevance and future perspectives}

Rare variants that arise in a Mega-NUMT will be at very low allelic fractions unless propagated into multiple copies of the $16.6 \mathrm{~kb}$ repeat. However, deletions and inversions may be more problematic: tandem repeats are prone to rearrangement and such events were noted in two of the studies 8,10 . In clinical genetics and other disciplines where it is important to clarify whether a multiHet pattern is due to rare Mega-NUMT or genuine mtDNA variants, muscle DNA should be used if possible. This procedure is relatively routine in adult mitochondrial disease clinics where biopsies are frequently performed for respiratory chain enzyme assays. In other situations, a cost-effective way to resolve unexpectedly high levels of heteroplasmy is to analyse DNA from hair shafts. Establishing a Mega-NUMT database that contains genomic positions and the mtDNA haplotype involved will also be of great use to disentangle future cases of multiHet individuals. Additional information should also include the range of repeat numbers seen, secondary rearrangements associated with the Mega-NUMT and validated PCR primers that can be used to test for such integrations.

Further studies on Mega-NUMTs will determine how often such rearrangements occur de novo and whether they can lead to disease, most likely via disruption of a constrained nuclear gene. The precise structure of Mega-NUMTs can be determined using a combination of Southern blotting and ultra-long-read sequencing methodologies. Published data regarding the number of repeat units, ranging from $2-56^{8,9}$, are indirect estimates rather than direct measurements. More precise comparisons of Mega-NUMTs with identical insertion sites will shed light on the evolution and approximate ages of these complex structural rearrangements. Other unanswered questions include how Mega-NUMTS occur and whether there is an RNA intermediate. Is there a two-stage process involving integration of a singlecopy NUMT and subsequent amplification, or a single insertion event? The circular nature of mtDNA makes the latter option combined with a rolling-circle mechanism seem attractive. Further work will help improve the understanding of replicative and transcriptional dynamics 
within mitochondria, and whether there are any common sequence motifs found at insertions sites akin to L1-mediated insertions, which are enriched for AATTTT.

The provocative study by Luo et al. has prompted detailed follow-up studies from scientists working in the fields of genomics, forensics and commercial clinical genetics testing. This has led to a diverse set of experimental data supporting the existence of mega-NUMTs more quickly than might have occurred otherwise, providing an alternative explanation that does not challenge established dogma, and advances our understanding of the evolving human genome.

1. Giles, R. E., Blanc, H., Cann, H. M. \& Wallace, D. C. Maternal inheritance of human mitochondrial DNA. Proc Natl Acad Sci U S A 77, 6715-6719 (1980).

2. Schwartz, M. \& Vissing, J. Paternal inheritance of mitochondrial DNA. N Engl J Med 347, 576580 (2002).

3. Luo, S. et al. Biparental Inheritance of Mitochondrial DNA in Humans. Proc Natl Acad Sci U S A 115, 13039-13044 (2018).

4. McWilliams, T. G. \& Suomalainen, A. Mitochondrial DNA can be inherited from fathers, not just mothers. Nature 565, 296-297 (2019).

5. Salas, A., Schonherr, S., Bandelt, H. J., Gomez-Carballa, A. \& Weissensteiner, H. Extraordinary claims require extraordinary evidence in asserted mtDNA biparental inheritance. Forensic Sci Int Genet 47, 102274 (2020).

6. Balciuniene, J. \& Balciunas, D. A Nuclear mtDNA Concatemer (Mega-NUMT) Could Mimic Paternal Inheritance of Mitochondrial Genome. Front Genet 10, 518, doi:10.3389/fgene.2019.00518 (2019).

7. Rius, R. et al. Biparental inheritance of mitochondrial DNA in humans is not a common phenomenon. Genet Med 21, 2823-2826 (2019).

8. Wei, W. et al. Nuclear-mitochondrial DNA segments resemble paternally inherited mitochondrial DNA in humans. Nat Commun 11, 1740 (2020).

9. Lutz-Bonengel, S. et al. Evidence for multi-copy Mega-NUMTs in the human genome. Nucleic Acids Res 49, 1517-1531 (2021).

10. Bai, R. et al. Interference of nuclear mitochondrial DNA segments in mitochondrial DNA testing resembles biparental transmission of mitochondrial DNA in humans. Genet Med, doi:10.1038/s41436-021-01166-1 (2021). 


\section{Acknowledgements}

The authors acknowledge funding from the Wellcome Trust (203141/Z/16/Z)\& (212219/Z/18/Z), the Medical Research Council Mitochondrial Biology Unit (MC_UU_00015/9), and the NIHR Biomedical Research Centres at Cambridge, Oxford and Great Ormond Street.

\section{Competing interests}

The authors declare no competing interests. 